TITLE:

\title{
Perfusion CT is superior to angiography in predicting pancreatic necrosis in patients with severe acute pancreatitis.
}

\section{AUTHOR(S):}

Tsuji, Yoshihisa; Hamaguchi, Kyoko; Watanabe, Yuji; Okumura, Akira; Isoda, Hiroyoshi; Yamamoto, Naoki; Kikuchi, Osamu; ... Togashi, Kaori; Yamamoto, Hiroshi; Chiba, Tsutomu

\section{CITATION:}

Tsuji, Yoshihisa ... [et al]. Perfusion CT is superior to angiography in predicting pancreatic necrosis in patients with severe acute pancreatitis.. Journal of gastroenterology 2010, 45(11): 1155-1162

\section{ISSUE DATE:}

2010-11

URL:

http://hdl.handle.net/2433/135421

\section{RIGHT:}

The final publication is available at www.springerlink.com; この論文は 出版社版でありません。引用の際には出版社版をご確認ご利用くださ $\omega_{\circ}$; This is not the published version. Please cite only the published version. 
Title: Perfusion CT is superior to angiography in predicting pancreatic necrosis in patients with severe acute pancreatitis

Short Title: Pancreatic perfusion CT and angiography

${ }^{* \dagger}$ Yoshihisa Tsuji, M.D., ${ }^{\dagger}$ Kyoko Hamaguchi, M.D., ${ }^{\dagger \dagger}$ Yuji Watanabe, M.D., ${ }^{\dagger \dagger}$ Akira Okumura, M.D., "Hiroyoshi Isoda, M.D., ${ }^{\dagger}$ Naoki Yamamoto, M.D., ${ }^{\dagger}$ Osamu Kikuchi, M.D., ${ }^{\dagger \dagger}$ Hiroyuki Yamamoto, R.T., ${ }^{\dagger}$ Kazuhiro Matsueda, M.D., ${ }^{*}$ Kenji Ueno, M.D., "Shinsuke Tada, M.D., " Kaori Togashi, M.D., PhD., ${ }^{\dagger}$ Hiroshi Yamamoto, M.D., and ${ }^{\star} T$ sutomu Chiba, M.D., PhD.

${ }^{\dagger}$ Department of Gastroenterology, Kurashiki Central Hospital, Okayama, Japan ${ }^{\dagger \dagger}$ Department of Radiology, Kurashiki Central Hospital, Okayama, Japan *Department of Gastroenterology and Hepatology, Kyoto University Graduate School of Medicine, Kyoto, Japan

**Department of Diagnostic Imaging and Nuclear Medicine, Kyoto University Graduate School of Medicine, Kyoto, Japan

How each author was involved; Data was analyzed by Yoshihisa Tsuji, Kyoko Hamaguchi, Yuji Watanabe, Akira Okumura, Hiroyoshi Isoda, Naoki Yamamoto, Osamu Kikuchi, Hiroyuki Yamamoto, Kazuhiro Matsueda, Kenji Ueno, Shinsuke Tada, and Kaori Togashi. Study concept was defined by Yoshihisa Tsuji, Hiroshi Yamamoto and Tsutomu Chiba. Yoshihisa Tsuji is conductor of this study. 
Grant support: Japan Society for the promotion science

Abbreviations used in this paper: CT, computed tomography; P-CT, perfusion CT; CECT, contrast-enhanced CT; SAP, severe acute pancreatitis; H.U, Hounsfield unit

Conflict of interest: All authors declare that they are free of any commercial affiliations or other financial interests that might lead to the appearance of conflict of interest.

Correspondence to: Tsutomu Chiba, M.D. PhD, Department of Gastroenterology and Hepatology, Kyoto University Graduate School of Medicine, Shogoinkawara-cho 54, Sakyo-ku, Kyoto, Japan

Phone: +81-75-751-4302; fax: +81-75-751-4303 


\section{Abstract}

Background: We performed perfusion CT (P-CT) and angiography of the pancreas in patients with severe acute pancreatitis (SAP) and compared the usefulness of these two methods in predicting the development of pancreatic necrosis.

Methods: We compared P-CT and angiography results taken within three days after symptoms onset in 21 SAP patients. We divided the pancreas into three areas, the head, body and tail, and examined each area for perfusion defects (via P-CT) and the arterial vasospasms (by angiography). Three weeks later, all patients underwent contrast-enhanced CT to confirm the development of pancreatic necrosis.

Results: Of the 21 SAP patients, 16 exhibited perfusion defects, while 17 proved positive for vasospasms in at least one area. Fourteen patients developed pancreatic necrosis. Of the 63 pancreatic areas from 21 SAP patients, perfusion defects appeared in 25 areas (39.7\%), 24 of which showed vasospasms (96.0\%). Angiography showed 33 areas with vasospasms (52.4\%), of which 24 showed perfusion defects (72.7\%). Of the 25 areas with perfusion defects, 21 developed pancreatic necrosis (84.0\%). Of the 33 areas with vasospasms, 21 developed 
necrosis (63.6\%). Pancreatic necrosis developed only in the areas positive both for perfusion defects and for vasospasms. No areas without perfusion defect or vasospasms developed pancreatic necrosis. P-CT predicted the development of pancreatic necrosis with significantly higher accuracy than angiography.

Conclusion: While both P-CT and angiography are useful in predicting the development of pancreatic necrosis, P-CT appears to be superior in it prediction in patients with SAP.

Key words: pancreatic necrosis; perfusion CT; angiography 


\section{Introduction}

Despite recent improvements in the treatment of severe acute pancreatitis (SAP), mortality rates remain high ${ }^{1}$, reaching as high as $20 \%$ in Japan. $^{2}$ SAP often leads to necrotizing pancreatitis, ${ }^{2}$ the most severe form of pancreatitis, that results from pancreatic ischemia caused by vasospasms of the intra-pancreatic arteries. $^{3}$ Accordingly, early detection of vasospasms in the intra-pancreatic arteries is critical in successfully treating SAP and improving prognosis. ${ }^{4,5} \mathrm{At}$ present, vasospasms of the intra-pancreatic arteries can only be diagnosed accurately by angiography. However, neither unstable nor emergent cases lend themselves to examination by angiography: thus, an alternative non-invasive method for diagnosing pancreatic ischemia is required.

We recently reported that perfusion CT is capable of detecting pancreatic perfusion defects non-invasively, and accurately predict the development of pancreatic necrosis at the early stages of SAP., ${ }^{6}$ Since vasospasms of the intra-pancreatic arteries are likely to cause perfusion defects in the pancreas, it might appear reasonable to assume that perfusion defects detected by perfusion CT reflect vasospasms of the intra-pancreatic arteries. However, no reports to date have examined the relationship between pancreatic perfusion defects 
detected by perfusion $\mathrm{CT}^{6,8-11}$ and vasospasms of the intra-pancreatic arteries as diagnosed by angiography. ${ }^{3-5}$

In this study, we examined the relationship between the results of pancreatic perfusion defects detected by perfusion CT and those of vasospasms of the intra-pancreatic arteries diagnosed by angiography in patients with SAP. We then compared the usefulness of the two methods in predicting the development of pancreatic necrosis. 


\section{Methods}

\section{Patients}

The study population consisted of 21 consecutive adult patients with SAP admitted to the department of gastroenterology at Kurashiki Central Hospital, Kurasiki, or Kyoto University Hospital, Kyoto, Japan, within three days of the onset of symptoms during the period from 2003 through 2008. A diagnosis of acute pancreatitis was based on physical findings of acute abdominal pain, laboratory findings of abnormal elevated serum amylase and serum lipase levels (more than three times above normal), and radiographic (CT) findings of pancreatitis $^{12,13}$ (Figures $1 \mathrm{~A}$ and 2A). SAP was diagnosed by Atlanta classification. ${ }^{14,15}$ After patients were diagnosed with SAP in the emergency room, perfusion CT was performed. Angiography was performed following perfusion CT. The study excluded patients in whom acute pancreatitis was related to cancer or a side effect of endoscopic retrograde cholangiopancreatography.

All patients were treated with fluid resuscitation in the intensive care unit to maintain central venous pressures above $8 \mathrm{mmHg}$. The patients were also administered a continuous regional arterial infusion therapy of prophylactic 
antibiotics (TIENAM®; Imipenem/cilastatin, BANYU PHARMACEUTICAL CO., LTD, Tokyo, Japan) with anticoagulation factors (FUTHAN®; nafamostat mesilate, TORII PHARMACEUTICAL CO., LTD, Tokyo, Japan) for 5 days. 16-18 To perform regional arterial infusion therapy, the tips of the catheters were placed at both celiac trunk and superior mesenteric artery. Patients who were free of severe ileus were administered enteral feedings through a nasojejunal tube. $^{19}$

\section{Study protocol}

We evaluated sequentially-performed perfusion CT and angiographies of 21 patients with SAP, dividing the pancreas into three areas (the head, body, and tail) and examining each area for perfusion defects by perfusion CT and arterial vasospasms by angiography (Figures 1 and 2). The development of pancreatic necrosis was confirmed by conventional contrast-enhanced CT (CECT) at three weeks following onset. The study protocol was approved by the Ethics Committee of each institution.

\section{Perfusion CT protocol}


A 16-slice or 64-slice helical CT scanner (Toshiba Aquillion $16 \AA$ or Toshiba Aquillion 64®, Toshiba Medical Systems Co., Ltd., Tochigi, Japan) was used. After obtaining transaxial images of the upper abdomen, we selected four sections (slice thickness of $5.0 \mathrm{~mm}$ ), covering as much of the pancreas as possible. As previously described, ${ }^{7}$ perfusion CT was performed after a bolus injection of $600 \mathrm{mgl} / \mathrm{kg}$ of contrast matter (Iomeron®; iomeprol, Eisai Co., Ltd., Tokyo, Japan ) of 10 seconds duration into a cubital vein [ $80 \mathrm{kVp}, 40 \mathrm{~mA}, 1.5$ s/rot, $60 \mathrm{sec}$, radiological exposure; $63.8 \pm 4.7 \mathrm{mGy}(\mathrm{CT} \text { dose index) }]^{20}$

For each pixel, changes in CT value were observed and a time-density curve obtained. ${ }^{6,21}$ Commercially-available software (box-MTF, Ziosoft, Inc., Tokyo, Japan) was then used to analyze the time-density curve. We calculated the pancreatic blood flow at each pixel by perfusion CT, as previously described. ${ }^{6,7,22,23}$ The deconvolution method was adjusted to enable analysis of pancreatic blood flow, which was then displayed as color maps..$^{6,74-26}$ The deconvolution method required placement of regions of interest on one artery and one vein to generate arterial input functions and venous outflow functions, respectively. ${ }^{24,27-29}$ In this study, the splenic artery was regarded as input and the splenic vein as outflow. $6,7,30$ 
In perfusion $\mathrm{CT}$, the color scale appears as a bar on the left edge of the perfusion images (Figures $1 \mathrm{~B}$ and $2 \mathrm{~B}$ ). The red areas in the perfusion images indicate faster blood flow (>150 $\mathrm{ml} / 100 \mathrm{~g} / \mathrm{min}$ ), while purple areas indicate slower blood flow $(<5 \mathrm{ml} / 100 \mathrm{~g} / \mathrm{min}){ }^{6,7}$

\section{Diagnosis of pancreatic perfusion defect by perfusion CT}

We made a diagnosis of pancreatic perfusion defect by perfusion CT as previously described. ${ }^{6,7}$ In brief, if the color map on perfusion CT demonstrated homogeneously high pancreatic blood flow within the entire pancreatic area, the area was determined to be negative for pancreatic perfusion defects (Figure 1B). If the color map on perfusion CT demonstrated partial pancreatic perfusion defect with pancreatic blood flow slower than hepatic blood flow (Figure 2B, red arrow), the area was defined to be positive for a pancreatic perfusion defect.

\section{Diagnosing vasospasms of the intra-pancreatic arteries}

We applied angiography to examine the celiac artery, supra mesenteric artery, and branches for arterial vasospasms. ${ }^{3,4}$ Vasospasms of the arteries were defined as luminal narrowing of $<50 \%$ (Figure $2 \mathrm{C}$ ).

\section{Diagnosing pancreatic necrosis}


The final diagnosis of pancreatic necrosis was made by performing conventional CECT at three weeks following symptom onset (Figures 1D and $1 \mathrm{E}){ }^{28}$

\section{Statistical analysis}

The unadjusted relationship between areas with perfusion defects and areas with vasospastic arteries was assessed by chi-square analysis. The unadjusted relationship between the results of prediction for development of pancreatic necrosis by perfusion CT and by angiography was also assessed by chi-square analysis. $P$ values below 0.05 were regarded as statistically significant. All statistical analysis was performed using SPSS 11 (SPSS Inc., Chicago, IL, USA). 


\section{Results}

Twenty-one SAP patients underwent both perfusion CT and angiography in a sequential manner within three days following the onset of symptoms. The average age and APACHE II score of the SAP patients were $53.2 \pm 13.2$ and $12.0 \pm 6.8$ (mean \pm standard deviation), respectively (Table 1 ). Of the 21 patients, 14 patients developed pancreatic necrosis, as diagnosed by conventional CECT performed three weeks following onset. Four of these SAP patients died (Table 1). Causes of death included infectious endocarditis, multiple organ failure due to infected pancreatic necrosis, multiple organ failure with systemic infection, and non-occlusive mesenteric ischemia (Table 1).

Of the 21 SAP patients, 16 were diagnosed as positive for pancreatic perfusion defects by perfusion CT in at least one area, while five were diagnosed as negative in all areas. On the other hand, of the 21 patients, 17 were diagnosed as positive in at least one area for vasospasms of the intra-pancreatic arteries by angiography, while four were negative in all areas (Table 1).

Of the 63 pancreatic areas (head, body, and tail) analyzed in the 21 SAP patients, seven occurred in the head areas, 12 in the body areas, and six in the tail areas, for a total of 25 areas (39.7\%) with perfusion defects. Eight head 
areas, 16 body areas, and nine tail areas for a total of 33 areas (52.4\%) were diagnosed with vasospastic arteries by angiography (Table 1).

Of the 25 areas with perfusion defects detected by perfusion CT, 24 areas were positive for vasospastic arteries (96.0\%) and only one negative. Of the remaining 38 areas without perfusion defects, nine areas were positive and 29 areas negative for vasospastic arteries (Table 2). Of the 33 areas with vasospastic arteries, 24 were positive (72.7\%) and nine negative for perfusion defects by perfusion CT. Of the remaining 30 areas without vasospastic arteries, only one area was positive for perfusion defects. Thus, a diagnosis of the presence or absence of perfusion defects by perfusion CT and the presence or absence of vasospastic arteries by angiography was concordant for $53(84.1 \%)$ of the 63 areas (Table 2).

Of the 25 areas with perfusion defects as determined by perfusion CT, 21 finally developed pancreatic necrosis (84.0\%). Of the 33 areas with vasospastic arteries as determined by angiography, 21 developed pancreatic necrosis (63.6\%). Notably, pancreatic necrosis developed only in areas positive for both perfusion defects and vasospasms of the intra-pancreatic arteries. None of the areas free of either perfusion defects or vasospasms developed pancreatic 
necrosis (Table 2). Thus, both perfusion CT and angiography successfully predicted the development of pancreatic necrosis at statistically significant levels $(P<0.01$ and $P<0.05$, respectively) (Tables 2 and 3$)$.

While perfusion CT and angiography showed the same sensitivity (100\% vs. $100 \%$ ) and negative predictive values (NPV; $100 \%$ vs. 100\%) in predicting the development of pancreatic necrosis, perfusion CT showed higher specificity (90.4\% vs. $71.4 \%$ ) and a higher positive predictive value (PPV; $84.0 \%$ vs. $63.6 \%$, with a positive finding under perfusion CT associated with a higher likelihood for developing pancreatic necrosis than areas identified by angiography (10.5 vs. 3.5) (Table 3). The accuracy of perfusion CT in predicting the development of pancreatic necrosis was significantly greater than for angiography (93.7\% vs. $81.0 \%, p<0.05)$ (Table 3). 


\section{Discussion}

Pancreatic necrosis is a serious complication of SAP that determines patient prognosis. $^{31,32}$ To improve the prognosis for SAP, it is crucial to predict the development of necrosis early and to initiate treatment as early as possible. Pancreatic necrosis results from pancreatic ischemia caused by vasospasms of the intra-pancreatic arteries. ${ }^{3-5}$ While angiography is currently the only established method for detecting vasospasms of the intra-pancreatic arteries, ${ }^{3,4}$ angiography is often difficult to perform in cases of unstable or emergent patients. In response to these drawbacks, we recently applied perfusion CT for patients with SAP. We found perfusion CT useful in the early detection of ischemic change in the pancreas. Here, we compared usefulness of angiography and perfusion CT in predicting the development of pancreatic necrosis in 21 patients with SAP, and found that both perfusion CT and angiography are useful for predicting development of pancreatic necrosis, but perfusion CT is superior to angiography for prediction.

Our study divided the whole pancreas into three areas, the head, body, and tail, and evaluated the results of perfusion CT and angiography for each area. Our examination showed 24 areas positive for both perfusion defects by 
perfusion CT and vasospasms by angiography and 29 areas negative by either method. Thus, the results of perfusion CT and angiography were concordant in $84.1 \%$ of all pancreatic areas from the 21 patients. The data indicates a high correlation between perfusion defects detected by perfusion CT and vasospasms of the intra-pancreatic arteries detected by angiography. However, it is worth noting that areas with vasospastic arteries clearly outnumber areas with perfusion defects (33 vs. 25); Moreover, although not all areas positive for vasospastic arteries of the intra-pancreatic arteries by angiography were positive for perfusion defects, most areas with perfusion defects by perfusion CT were positive for vasospasms of the intra-pancreatic arteries, potentially suggesting that perfusion defects detected by perfusion CT in most cases result from vasospasms of the intra-pancreatic arteries. In a finding that supports this idea, Inoue et al. reported that endothelin-1, which can produce spastic change in the arteries, is an important mediator in the development of pancreatic ischemia in cases of severe acute pancreatitis. ${ }^{33}$

Our study found both perfusion CT and angiography useful in predicting the development of pancreatic necrosis. Indeed, sensitivity and NPV were $100 \%$ for both methods, and specificities were $90.4 \%$ and $71.4 \%$, respectively, for 
perfusion CT and angiography. The angiography data in this study was comparable to data from previous studies, ${ }^{3,4}$ while the perfusion CT data was comparable to data from our previous study. ${ }^{7}$ An important finding of the present study is that $84.0 \%$ of the areas with perfusion defects developed pancreatic necrosis; this was true of only $63.6 \%$ of the areas with vasospastic arteries. Moreover, none of the areas negative for both perfusion defects or vasospastic arteries developed pancreatic necrosis. In short, perfusion CT proved more accurate in predicting the development of pancreatic necrosis than angiography.

The reasons why the discrepancy exists between angiography and perfusion CT and why perfusion CT is superior to angiography for predicting pancreatic necrosis in patients with SAP are unknown at present. It has been shown that perfusion CT can diagnose microcirculation failure ${ }^{24,29,34,35}$. In contrast, angiography can diagnose spastic lesions of large arteries, which might not always result in impairment of microcirculation. Thus, our data might suggest that vasospasm of large arteries does not always result in impairment of microcirculation. In any event, our present data may suggest that disturbance of microcirculation detected by perfusion CT is a critical event for development of pancreatic necrosis, and thus, perfusion CT is superior to angiography for 
predicting development of pancreatic necrosis.

We should emphasize that pancreatic necrosis developed only in areas positive for both perfusion CT and angiography, and none of the areas negative by either method developed necrosis in this study. Therefore, patients positive under both perfusion CT and angiography would appear to be at very high risk for pancreatic necrosis. In contrast, a negative result for either perfusion CT or angiography alone might rule out the future development of necrosis. This data is consistent with our previous study, ${ }^{7}$ which showed that 9 of 10 patients in whom perfusion defects were detected by perfusion CT developed pancreatic necrosis, whereas necrosis did not develop in any patient in whom no perfusion defects were detected. The data is also in agreement with the report by Takeda et al. showing that by analyzing 147 patients with acute necrotizing pancreatitis no pancreatic tissue with the absence of vasospasms in the intra-pancreatic arteries developed pancreatic necrosis. ${ }^{3}$

As previously reported, prognosis of acute necrotizing pancreatitis ${ }^{1-3}$ and that of ischemic acute pancreatitis ${ }^{4-9}$ are generally poor. However, the prognosis of our SAP patients with vasospasm or ischemia was not so poor in this study. Although the reason for such different outcomes between our patients and those 
in previous reports is not clear at present, it may be emphasized that all of our cases were treated with regional arterial infusion therapy in early stage of SAP. Thus, it is possible that the prognosis of the SAP patients was influenced and improved by regional arterial infusion therapy in our study ${ }^{16-18}$, although whether or not regional arterial infusion therapy really improves prognosis of SAP patients with pancreatic ischemia should be elucidated in future studies. It may be noted that some cases who showed pancreatic ischemia by perfusion CT or vasospasm by angiography did not develop pancreatic necrosis (case 4, 5, 15 and 18-21) in this study. Although the reason for such discrepancy is not clear at present, again it may be possible that regional arterial infusion therapy may have prevented development of pancreatic necrosis in our study ${ }^{16-18}$.

There has been vigorous recent debate on treatment methods for SAP. For instance, the effectiveness of prophylactic antibiotics ${ }^{36-41}$ in cases of SAP remains an open question. Some reports argue for the efficacy of prophylactic antibiotics, ${ }^{41}$ while others reach the opposite finding. ${ }^{39,40}$ If the goal of a study is to determine whether administering prophylactic antibiotics prevents pancreatic necrosis, the most severe manifestation of SAP, antibiotics must, of course, be administered before pancreatic necrosis actually develops. Indeed, Besselink et 
al. reported that in their cohort study infection occurred early in acute pancreatitis and had a significant impact on mortality, suggesting that prophylactic strategies should be examined as early intervention. ${ }^{41}$ In this regard, all previous studies have involved the administration of antibiotics to patients in whom pancreatic necrosis had already been diagnosed. ${ }^{39-41}$ As we demonstrate in this paper, perfusion CT offers a safe and accurate route to diagnosing pancreatic ischemia, a precondition of pancreatic necrosis. Thus, by enabling the diagnosis of pancreatic ischemia, perfusion CT should prove useful in studies investigating effectiveness of the administration of antibiotics as a prophylactic measure.

In conclusion, this study found both perfusion CT and angiography useful in predicting the development of pancreatic necrosis, although perfusion CT proved more accurate. Perfusion CT, moreover, is less invasive and more readily available than angiography. Further study is needed to ascertain whether perfusion CT can substitute for angiography as a primary screening method when evaluating the severity of acute pancreatitis. 


\section{Reference}

1. Sekimoto M, Takada T, Kawarada Y, Hirata K, Mayumi T, Yoshida M, et al.

JPN Guidelines for the management of acute pancreatitis: epidemiology,

etiology, natural history, and outcome predictors in acute pancreatitis. J

Hepatobiliary Pancreat Surg 2006;13:10-24.

2. Beger HG, Isenmann R. Diagnosis, objective assessment of severity, and management of acute pancreatitis. Santorini Consensus Conference. Int J Pancreatol 1999;26:1-2; discussion 2-3.

3. Takeda K, Mikami Y, Fukuyama S, Egawa S, Sunamura M, Ishibashi T, et al. Pancreatic ischemia associated with vasospasm in the early phase of human acute necrotizing pancreatitis. Pancreas 2005;30:40-9.

4. Inoue K, Hirota M, Beppu T, Ishiko T, Kimura Y, Maeda K, et al. Angiographic features in acute pancreatitis: the severity of abdominal vessel ischemic change reflects the severity of acute pancreatitis. Jop 2003;4:207-13.

5. Cuthbertson CM, Christophi C. Disturbances of the microcirculation in acute pancreatitis. Br J Surg 2006;93:518-30. 
6. Tsuji Y, Watanabe Y, Matsueda K, Yamamoto H, Ishida E, Yamamoto H.

Usefulness of perfusion computed tomography for early detection of pancreatic ischemia in severe acute pancreatitis. J Gastroenterol Hepatol 2006;21:1506-8.

7. Tsuji Y, Yamamoto H, Yazumi S, Watanabe Y, Matsueda K, Yamamoto H, et al. Perfusion computerized tomography can predict pancreatic necrosis in early stages of severe acute pancreatitis. Clin Gastroenterol Hepatol 2007;5:1484-92.

8. Bize PE, Platon A, Becker CD, Poletti PA. Perfusion measurement in acute pancreatitis using dynamic perfusion MDCT. AJR Am J Roentgenol 2006;186:114-8.

9. Park MS, Klotz E, Kim MJ, Song SY, Park SW, Cha SW,et al. Perfusion CT: noninvasive surrogate marker for stratification of pancreatic cancer response to concurrent chemo- and radiation therapy. Radiology 2009;250:110-7.

10. Sheiman RG, Sitek A. Feasibility of measurement of pancreatic perfusion parameters with single-compartment kinetic model applied to dynamic contrast-enhanced CT images. Radiology 2008;249:878-82. 
11. d'Assignies G, Couvelard A, Bahrami S, Vullierme MP, Hammel P, Hentic O, et al. Pancreatic Endocrine Tumors: Tumor Blood Flow Assessed with Perfusion CT Reflects Angiogenesis and Correlates with Prognostic Factors. Radiology 2008.

12. Balthazar EJ. Staging of acute pancreatitis. Radiol Clin North Am 2002;40:1199-209.

13. Balthazar EJ, Freeny PC, vanSonnenberg E. Imaging and intervention in acute pancreatitis. Radiology 1994;193:297-306.

14. UK guidelines for the management of acute pancreatitis. Gut 2005;54 Suppl 3:iii1-9.

15. Bollen TL, van Santvoort HC, Besselink MG, van Leeuwen MS, Horvath KD, Freeny PC, et al. The Atlanta Classification of acute pancreatitis revisited. $\mathrm{Br}$ J Surg 2008;95:6-21.

16. Takeda K, Yamauchi J, Shibuya K, Sunamura M, Mikami Y, Matsuno S. Benefit of continuous regional arterial infusion of protease inhibitor and antibiotic in the management of acute necrotizing pancreatitis. Pancreatology 2001;1:668-73. 
17. Takeda K, Matsuno S, Sunamura M, Kakugawa Y. Continuous regional arterial infusion of protease inhibitor and antibiotics in acute necrotizing pancreatitis. Am J Surg 1996;171:394-8.

18. Takeda K. Antiproteases in the treatment of acute necrotizing pancreatitis: continuous regional arterial infusion. Jop 2007;8:526-32.

19. Eckerwall GE, Axelsson JB, Andersson RG. Early nasogastric feeding in predicted severe acute pancreatitis: A clinical, randomized study. Ann Surg 2006;244:959-65; discussion 965-7.

20. Tsuji. Y, Koizumi. K, Isoda. H, Ueno. K, Tada. S, Chiba. T. The radiological exposure of pancreatic perfusion CT. pancreas 2009:in press.

21. Tsushima Y, Blomley JK, Kusano S, Endo K. The portal component of hepatic perfusion measured by dynamic CT: an indicator of hepatic parenchymal damage. Dig Dis Sci 1999;44:1632-8.

22. Miles KA, Hayball MP, Dixon AK. Measurement of human pancreatic perfusion using dynamic computed tomography with perfusion imaging. $\mathrm{Br} \mathrm{J}$ Radiol 1995;68:471-5. 
23. Tsushima Y, Kusano S. Age-dependent decline in parenchymal perfusion in the normal human pancreas: measurement by dynamic computed tomography. Pancreas 1998;17:148-52.

24. Abe H, Murakami T, Kubota M, Kim T, Hori M, Kudo M, et al. Quantitative tissue blood flow evaluation of pancreatic tumor: comparison between xenon CT technique and perfusion CT technique based on deconvolution analysis. Radiat Med 2005;23:364-70.

25. Wintermark M, Ko NU, Smith WS, Liu S, Higashida RT, Dillon WP. Vasospasm after subarachnoid hemorrhage: utility of perfusion CT and CT angiography on diagnosis and management. AJNR Am J Neuroradiol $2006 ; 27: 26-34$

26. Axel L. Cerebral perfusion CT techniques. Radiology 2004;233:935; author reply 935.

27. Eastwood JD, Lev MH, Azhari T, Lee TY, Barboriak DP, Delong DM, et al. CT perfusion scanning with deconvolution analysis: pilot study in patients with acute middle cerebral artery stroke. Radiology 2002;222:227-36. 
28. Eastwood JD, Provenzale JM, Hurwitz LM, Lee TY. Practical injection-rate CT perfusion imaging: deconvolution-derived hemodynamics in a case of stroke. Neuroradiology 2001;43:223-6.

29. Sahani DV, Holalkere NS, Kambadakone A, Matthes K, Mino-Kenudson M, Brugge WR. Role of computed tomography perfusion in the evaluation of pancreatic necrosis and pancreatitis after endoscopic ultrasound-guided ablation of the pancreas in a porcine model. Pancreas 2009 May 22.Epub ahead of print

30. Kishimoto M, Tsuji Y, Katabami N, Shimizu J, Lee KJ, Iwasaki T,et al. Measurement of canine pancreatic perfusion using dynamic computed tomography: Influence of input-output vessels on deconvolution and maximum slope methods. Eur J Radiol 2009 July 7. Epub ahead of print

31. Traverso LW, Kozarek RA. Pancreatic necrosectomy: definitions and technique. J Gastrointest Surg 2005;9:436-9.

32. Isenmann R, Rau B, Zoellner U, Beger HG. Management of patients with extended pancreatic necrosis. Pancreatology 2001;1:63-8. 
33. Inoue K, Hirota M, Kimura Y, Kuwata K, Ohmuraya M, Ogawa M. Further evidence for endothelin as an important mediator of pancreatic and intestinal ischemia in severe acute pancreatitis. Pancreas 2003;26:218-23.

34. Wintermark M, Thiran JP, Maeder P, Schnyder P, Meuli R. Simultaneous measurement of regional cerebral blood flow by perfusion CT and stable xenon CT: a validation study. Am J Neuroradiol 2001;22:905-14.

35. Tsushima Y, Kusano S. Age-dependent decline in parenchymal perfusion in the normal human pancreas: measurement by dynamic computed tomography. Pancreas 1998;17:148-52.

36. Bai Y, Gao J, Zou DW, Li ZS. Prophylactic Antibiotics in Acute Pancreatitis: Further High-Quality Trials Are Still Warranted. Am J Gastroenterol 2008;103:104-10.

37. Tsuji Y, Chiba T. Are Prophylactic Antibiotics Really Ineffective in Reducing the Risk of Pancreatic Necrosis? Am J Gastroenterol 2008;103:2145-6..

38. Manes G, Uomo I, Menchise A, Rabitti PG, Ferrara EC. Timing of antibiotics prophylaxis in acute pancreatitis; A controlled randomized study with meropenem. Am J Gastroenterol 2006;101:1348-53 
39. Jafri NS, Mahid SS, Idstein SR, Hornung CA, Galandiuk S. Antibiotic prophylaxis is not protective in severe acute pancreatitis: a systematic review and meta-analysis. Am J Surg 2009;197:806-13.

40. Wittau M, Hohl K, Mayer J, Henne-Bruns D, Isenmann R. The weak evidence base for antibiotic prophylaxis in severe acute pancreatitis. Hepatogastroenterology 2008;55:2233-7.

41. Besselink MG, van Santvoort HC, Boermeester MA, Nieuwenhuijs VB, van Goor $\mathrm{H}$, Dejong $\mathrm{CH}$, et al. Timing and impact of infections in acute pancreatitis. Br J Surg 2009;96:267-73. 
Figure 1 Severe acute pancreatitis without vasospasms of the intra-pancreatic arteries or pancreatic perfusion defects

Shown here are images from patient 2 in Table 2. The pancreas is homogeneously enhanced in contrast-enhanced CT (A). In perfusion CT (B), pancreatic blood flow is homogeneously high, and the pancreas is free of any areas with perfusion defects (red arrow). Angiography showed no vasospastic arteries in the pancreas. Mild diffuse staining of the pancreas was observed (White arrows) (C). Contrast-enhanced CT three weeks later showed no signs of pancreatic necrosis (D).

Figure 2 Severe acute pancreatitis with vasospasms of the intra-pancreatic arteries and pancreatic perfusion defects Shown here are images from patient 6 in Table 2. The pancreas is homogeneously enhanced in contrast-enhanced CT (A). Perfusion CT shows markedly slower blood flow (red arrows) in the pancreas body (B). Thus, this case was diagnosed as positive for perfusion defect. Angiography (C) shows the splenic artery (white arrow) and its branches (Blue arrows) were found to be positive for vasospasms in the pancreas head, body and tail. Contrast-enhanced CT three weeks later detected pancreatic necrosis in the body (D, yellow arrow), and viable tissue in the tail (the image was not included in this slice). 

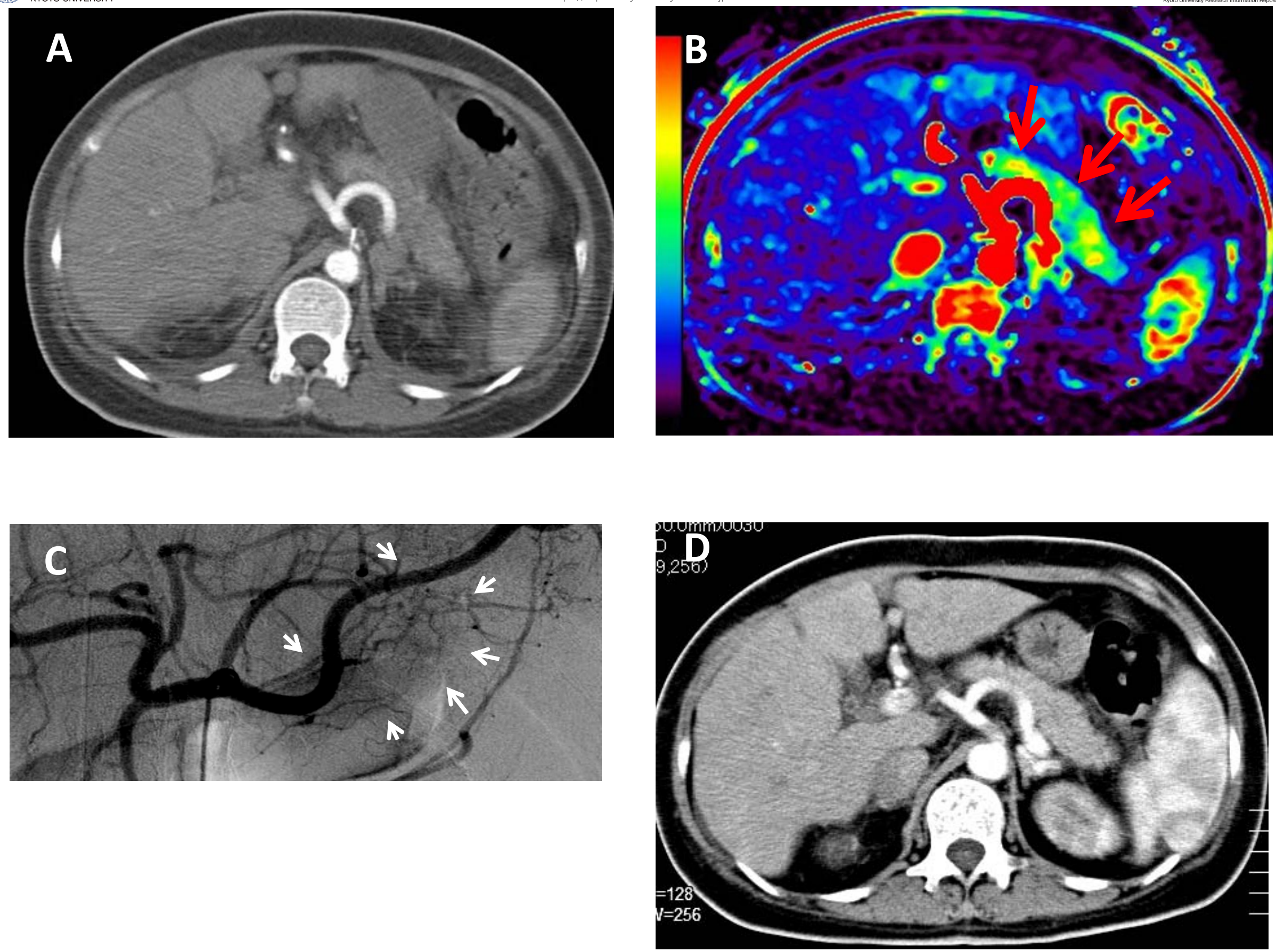
